\title{
Optimum Determination of Partial Transmission Ratios of Mechanical Driven Systems Using a Chain Drive and a Two-step Helical Gearbox
}

\author{
Vu Ngoc Pi, Tran Thi Phuong Thao and Dang Anh Tuan \\ Mechanical Engineering Faculty, Thai Nguyen University of Technology, Thai Nguyen 250000, Vietnam
}

\begin{abstract}
This paper presents a study on optimum determination of partial ratios of mechanical drive systems using a chain drive and two-step helical gearbox for getting minimum size of the system. The chosen objective function was the cross section dimension of the system. In solving the optimization problem, the design equation for pitting resistance of a gear set was investigated and equations on moment equilibrium condition of a mechanic system including a chain drive and two helical gear units and their regular resistance condition were analyses. From the results of the study, effective formulas for determination of the partial ratios of the chain drive and two-step helical gearboxes were introduced. As the formulas are explicit, the partial ratios can be calculated accurately and simply.
\end{abstract}

Key words: Gearbox design, optimum design, helical gearbox, chain drive, transmission ratio.

\section{Introduction}

Until now, there have been many studies on the prediction of the partial ratios of helical gearboxes. These studies were carried out on two-step gearboxes [1-4] and three-step gearboxes [2, 5, 6]. Also, many methods have been used in order to find the optimum partial ratios. These methods are the graph method [1, 2], the "practical method" [3] and modeling method [4-8]. However, all of the above researches were done for mechanical driven systems which do not use a belt or a chain drive. Recently, there have been several studies on determination of partial ratios of mechanical driven systems which use a V-belt and a gearbox [7, 8]. However, until now, there have not been studies for a system using a chain drive and a gearbox. This paper presents a study for optimum determination of partial ratios for mechanical driven systems using a chain drive and a two-step helical gearbox for getting the minimum system cross-sectional dimension.

Corresponding author: $\mathrm{Vu}$ Ngoc $\mathrm{Pi}$, Ph.D., associate professor, main research fields: optimum design of machine elements, abrasive machining.

\section{Theoretical Basis}

For a two-step helical gearbox (Fig. 1), the cross-sectional dimension is minimum when [1]:

$$
d_{w 21}=d_{w 22}
$$

From Eq. (1) and Fig. 1, it can be seen that, for a mechanical system which uses a chain drive and a two-step helical gearbox (Fig. 1), the cross-sectional dimension is minimum when:

$$
d_{w 21}=d_{w 22}=d_{2}
$$

In Eqs. (1) and (2), $d_{w 21}$ and $d_{w 22}$ are driven diameters of high and low speed of the gearbox; $d_{2}$ is the pitch diameter of the driven sprocket of the chain drive.

For a two-step helical gearbox, Eq. (1) is guaranteed when [4]:

$$
u_{2}=1.1 \cdot u_{g}^{1 / 3}
$$

In which, $u_{2}$ is transmission ratio of the low speed unit of the gearbox; $u_{g}$ is the transmission ratio of the gearbox; $u_{g}$ can be calculated by:

$$
u_{g}=u_{1} \cdot u_{2}=u_{t} / u_{c}
$$

Where, $u_{l}$ is transmission ratio of the high speed unit 

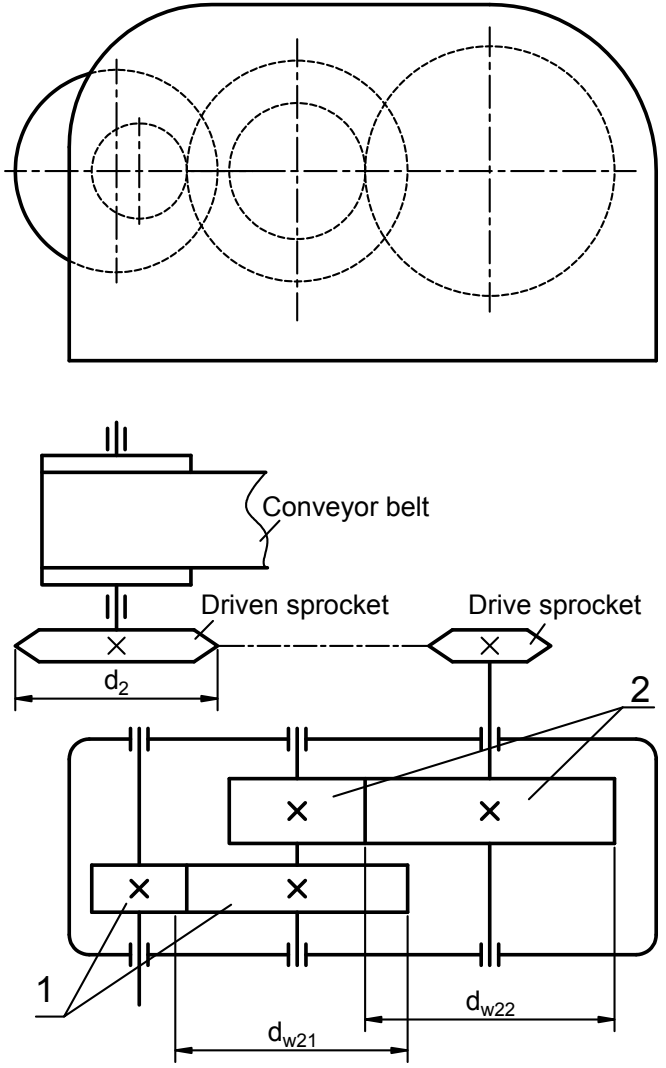

Fig. 1 Calculation chema.

of the gearbox; $u_{t}$ is the total transmission ratio of the system; $u_{c}$ is the transmission ratio of the chain drive.

From above analysis, for finding the optimum partial ratios of the systems in order to get the minimum system cross section, it is necessary to determine the diameters $d_{2}$ and $d_{w 22}$.

\subsection{Determining the Driven Sprocket Diameter $d_{2}$}

For the low step of the gearbox, Eq. (5) is used as the design equation for pitting resistance [9]:

$$
\sigma_{H 2}=Z_{M 2} \cdot Z_{H 2} \cdot Z_{\varepsilon 2} \cdot \sqrt{\frac{2 \cdot T_{12} \cdot K_{H 2} \cdot \sqrt{u_{2}+1}}{b_{w 2} \cdot d_{w 12}^{2} \cdot u_{2}}} \leq\left[\sigma_{H 2}\right]
$$

It follows from Eq. (5) that:

$$
\left[T_{12}\right]=\frac{b_{w 2} \cdot d_{w 12}^{2} \cdot u_{2}}{2 \cdot\left(u_{2}+1\right)} \frac{\left[\sigma_{H 2}\right]^{2}}{K_{H 2} \cdot\left(Z_{M 2} \cdot Z_{H 2} \cdot Z_{\varepsilon 2}\right)^{2}}
$$

where, $b_{w l}$ is the face width (mm) and $d_{w 11}$ is the pitch diameter of the first step; they are calculated by Eqs. (7) and (8):

$$
\begin{gathered}
b_{w 2}=\psi_{b a 2} \cdot a_{w 2}=\psi_{b a 2} \cdot d_{w 12} \cdot\left(u_{2}+1\right) / 2 \\
d_{w 12}=d_{w 22} / u_{2}
\end{gathered}
$$

When substituting Eqs. (7) and (8) into Eq. (6), authors get:

$$
\left[T_{12}\right]=\frac{\psi_{b a 2} \cdot d_{w 22}^{3} \cdot\left[K_{02}\right]}{4 \cdot u_{2}^{2}}
$$

in which

$$
\left[K_{02}\right]=\frac{\left[\sigma_{H 2}\right]^{2}}{K_{H 2} \cdot\left(Z_{M 2} \cdot Z_{H 2} \cdot Z_{\varepsilon 2}\right)^{2}}
$$

From Eq. (9), the pitch diameter $d_{w 22}$ can be calculated by:

$$
d_{w 22}=\left(\frac{4\left[T_{12}\right] u_{2}^{2}}{\psi_{b a 2}\left[K_{02}\right]}\right)^{1 / 3}
$$

\subsection{Determining the Driven Diameter $d_{22}$}

For a chain drive, the pitch diameter of the driven sprocket is calculated by Eq. (12) [9]:

$$
d_{2}=d_{1} \cdot u_{c}
$$

Where, $d_{l}$ is the pitch diameter of the drive sprocket. $d_{1}$ is determined by Eq. (13) [9]:

$$
d_{1}=p / \sin \left(\pi / z_{1}\right)
$$

In which,

$\mathrm{z}_{1}$-the number of teeth in the drive sprocket; From tabulated data for determining $\mathrm{z}_{1}$ [9], Eq. (14) was found for calculating $\mathrm{z}_{1}$ (with $R^{2}=0.995$ ):

$$
z_{1}=32.4-2.4 \cdot u_{c}
$$

$p$-the chain pitch (mm); $p$ is determined based on the design power capacity $\mathrm{P}$ which can be expressed Eq. (15) [9]:

$$
P=P_{1} \cdot k \cdot k_{z} \cdot k_{n}
$$

Where, $\mathrm{P}_{1}$ is the power rating $(\mathrm{kW})$ which can be calculated by: 

Systems Using a Chain Drive and a Two-Step Helical Gearbox

$$
P_{1}=\frac{T_{1} \cdot n_{1}}{9.55 \cdot 10^{6}}
$$

With $n_{1}$ is the revolution of the drive sprocket (rpm):

$$
\begin{gathered}
n_{1}=n_{m} / u_{g} \\
T_{1}=T_{o} \cdot \eta_{c} \cdot \eta_{b}
\end{gathered}
$$

In Eqs. (17) and (18), $\eta_{c}$ is efficiency of chain drive $\left(\eta_{c}=0.95-0.97\right.$ [9]); $\eta_{b}$ is efficiency of a pair of bearings $\left(\eta_{b}=0.99-0.995[9]\right) ; \mathrm{T}_{1}$ is the torque on the drive $(\mathrm{Nmm}) ; \mathrm{T}_{\mathrm{o}}$ is the output torque $(\mathrm{Nmm})$.

$k, k_{z}$ and $k_{n}$ are coefficients which are determined by Eqs. (19), (20) and (21):

$$
\begin{gathered}
k=k_{d} \cdot k_{p} \cdot k_{c} \cdot k_{a d j} \cdot k_{\text {lub }} \cdot k_{c o n} \\
k_{z}=25 / z_{1} \\
k_{n}=n_{01} / n_{1}
\end{gathered}
$$

In Eqs. (19), (20) and (21), $k_{d}$ is effect of shock factor; $k_{p}$ is effect of position of the drive; $k_{c}$ is effect of center distance; $k_{a d j}$ is effect of possibility of adjusting the center distance; $k_{l u b}$ is effect of lubrication; $k_{c o n}$ is effect of operating conditions; $n_{01}$ is tabulated number of teeth of the drive sprocket.

\section{Determining Partial Transmission Ratios}

For finding the partial ratios of the system, a computer program was built. The aim of the program is to find the optimum values of the transmission ratio of the chain drive $u_{c}$ which satisfies the condition (2). The chosen programming language was Matlab. The input values of the program were: $u_{t}=10 \div 220 ; u_{g}=2 \div 60$; $T_{o}=40,000 \div 2,000,000(\mathrm{Nmm}) ; k_{d}=1 ; k_{p}=1 ; k_{c}=1$; $k_{\text {adj }}=1 ; k_{\text {lub }}=1 ; k_{\text {con }}=1.25$.

Fig. 2 shows the relation between the optimum transmission ratio of the chain drive and the output torque. It was found that the optimum values of the

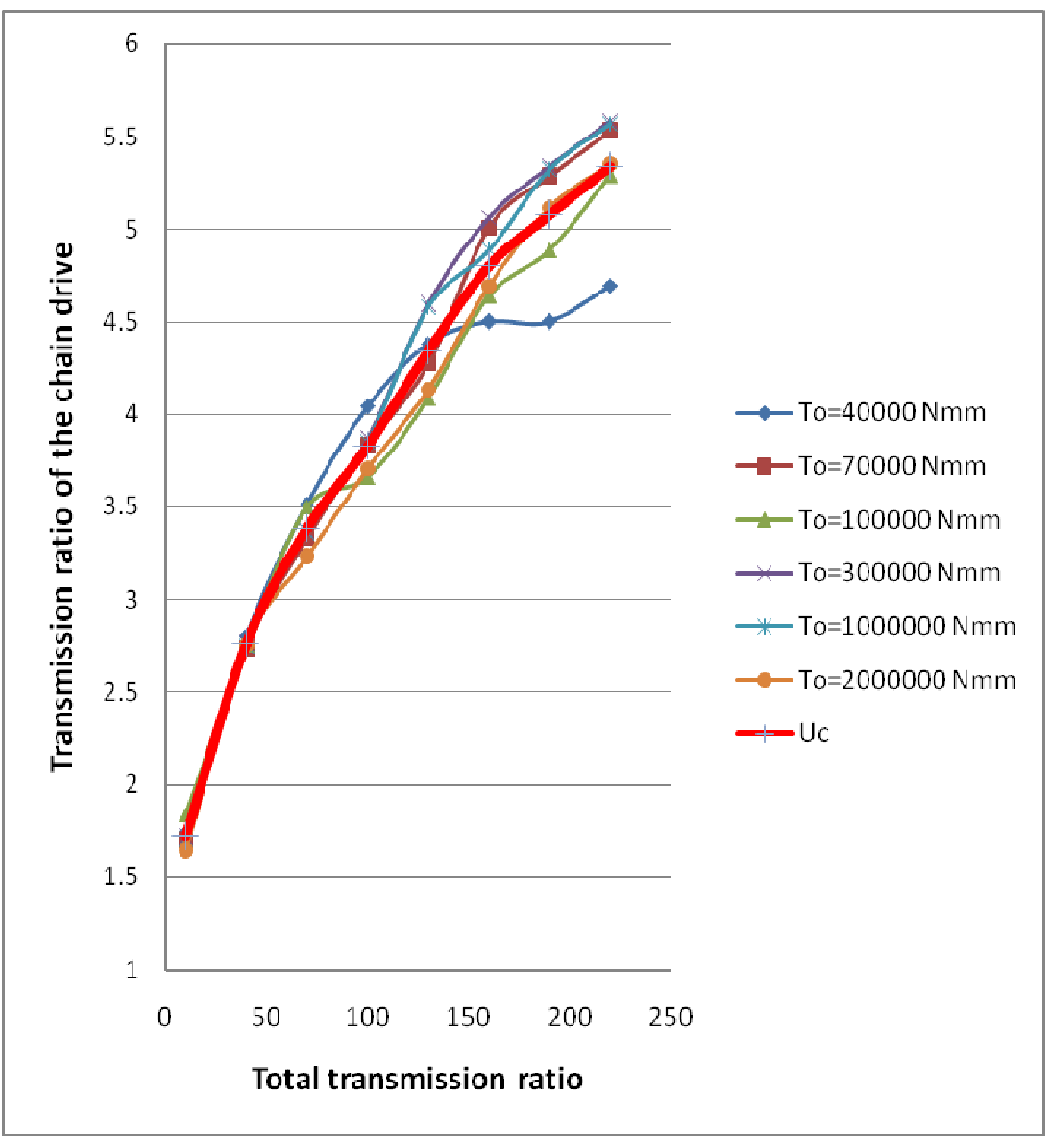

Fig. 2 Transmission ratio of the chain drive versus output torque. 
transmission ratio of the chain drive depend strongly on the total transmission ratio of the system (Fig. 2). Also, the output torque does not affect much on the transmission ratio of the chain drive. Therefore, the influence of the output torque can be replaced by choosing average values of the transmission ratio of the chain drive (the line of $\mathrm{u}_{\mathrm{c}}$ (Fig. 2)). In addition, Eq. (22) was found for determination of the optimum transmission ratio of the chain drive (with $\left.R^{2}=997\right)$ :

$$
u_{c}=0.718 \cdot u_{t}^{0.369}
$$

After finding the transmission ratio of the chain drive by Eq. (22), the partion ratios $u_{1}$ and $u_{2}$ of the gearbox can be easily found from Eqs. (3) and (4).

\section{Conclusion}

The minimum system cross-sectional dimension of a mechanical drive system using a chain drive and a two-step helical gearbox can be obtained by optimum splitting the total transmission ratio of the system.

A model for determining the optimum transmission ratio of the chain drive was proposed. Also, the partial transmission ratios of the two-step helical gearbox were found for getting the minimum cross-sectional dimension of the system.

By using explicit models, the partial transmission ratios of the chain drive and the gearbox can be determined accurately and simply.

\section{Acknowledgements}

The work described in this paper was supported by
Thai Nguyen University of Technology for a scientific project.

\section{References}

[1] Kudreavtev, V. N., Gierzaves, I. A., and Glukharev, E. G. 1971. "Design and Calculus of Gearboxes." Mashinostroenie Publishing, Sankt Petersburg.

[2] Petrovski, A. N., Sapiro, B. A., and Saphonova, N. K. 1987. "About Optimal Problem for Multi-step Gearboxes." Vestnik Mashinostroenie 10: 13-4.

[3] Milou, G., Dobre, G., Visa, F., and Vitila, H. 1996. "Optimal Design of Two-step Gear Units, regarding the Main Parameters." VDI Berichte 1230: 227-44.

[4] Pi, V. N. 2001. "A Method for Optimal Calculation of Total Transmission Ratio of Two Step Helical Gearboxes." Procedings of the National conference on Engineering Mechanics, Ha Noi, October 12-13.

[5] Pi, V. N., Binh, N. D., Dac, V. Q., and The, P. Q. 2006. "Optimal Calculation of Total Transmission Ratio of Three-step Helical Gearboxes for Minimum Mass of Gears." Journal of Science and Technology of 6 Engineering Universities 55: 91-3.

[6] Pi, V. N. 2008. "A New Study on Optimal Calculation of Partial Transmission Ratio of Three-step Helical Reducer." The 3rd IASME/WSEAS International Conference on Continuum Mechanics, Cambridge, UK, February 23-25.

[7] Pi, V. N., Thao, T. T. P., and Thao, L. T. P. 2015. "A New Study on Optimum Determination of Partial Transmission Ratios of Mechanical Driven Systems Using a V-belt and Two-step Helical Gearbox." Vietnam Mechanical Engineering Journal 10: 123-5.

[8] Pi, V. N., Cam, N. T. H., and Tuan, N. K. 2016. "Optimum Calculation of Partial Transmission Ratios of Mechanical Driven Systems Using a V-belt and Two-step Bevel Helical Gearbox." Journal of Environmental Science and Engineering A 5: 566-9.

[9] Trinh, C., and Uyen, L. V. 1998. Design and Calculus of Mechanical Transmissions. Educational Republishing House, Hanoi. 